\title{
Validation of tungsten cross sections in the neutron energy region up to $100 \mathrm{keV}$
}

\author{
Marco T. Pigni ${ }^{1, a}$, Gašper Žerovnik ${ }^{2}$, Luiz. C. Leal ${ }^{3}$, and Andrej Trkov ${ }^{4}$ \\ 1 Oak Ridge National Laboratory, PO Box 2008, Oak Ridge, TN, USA \\ 2 EC-JRC Geel, Belgium \\ 3 Institut de Radioprotection et de Sûreté Nucléaire, 92260 Fontenay-aux-Roses, France \\ 4 International Atomic Energy Agency, Nuclear Data Section, Austria
}

\begin{abstract}
Following a series of recent cross section evaluations on tungsten isotopes performed at Oak Ridge National Laboratory (ORNL), this paper presents the validation work carried out to test the performance of the evaluated cross sections based on lead-slowing-down (LSD) benchmarks conducted in Grenoble. ORNL completed the resonance parameter evaluation of four tungsten isotopes $-{ }^{182,183,184,186} \mathrm{~W}$ - in August 2014 and submitted it as an ENDF-compatible file to be part of the next release of the ENDF/B-VIII.0 nuclear data library. The evaluations were performed with support from the US Nuclear Criticality Safety Program in an effort to provide improved tungsten cross section and covariance data for criticality safety sensitivity analyses. The validation analysis based on the LSD benchmarks showed an improved agreement with the experimental response when the ORNL tungsten evaluations were included in the ENDF/B-VII.1 library. Comparison with the results obtained with the JEFF-3.2 nuclear data library are also discussed.
\end{abstract}

\section{Introduction}

Tungsten is a key structural element in process facilities and is present in several benchmark evaluations of the International Handbook of Evaluated Criticality Safety Benchmark Experiments (ICSBEP). Improved nuclear data evaluations for tungsten isotopes are important because of the long-standing computational bias associated with tungsten in benchmark calculations and attributed to the poor performance of the neutron capture cross sections for the stable tungsten isotopes, ${ }^{182,183,184,186} \mathrm{~W}$, in the ENDF/B-VII.0 [1]. Although in the ENDF/B-VII.1 library [2] much work was devoted to the improvement in the performance of criticality benchmarks containing tungsten isotopes, in some cases, by reducing the discrepancy by more than a factor of two, the results still showed a disagreement of about $700 \mathrm{pcm}$.

With support from the US Nuclear Criticality Safety Program (NCSP) in an effort to provide improved tungsten cross sections and covariance data for criticality safety sensitivity and uncertainty analyses, The Oak Ridge

\footnotetext{
a e-mail: pignimt@ornl .gov. Notice: This manuscript has been authored by UT-Battelle, LLC under Contract No. DE-AC0500OR22725 with the U.S. Department of Energy. The United States Government retains, the publisher, by accepting the article for publication, acknowledges that the United States Government retains a non-exclusive, paid-up, irrevocable, worldwide license to publish or reproduce the published form of this manuscript, or allow others to do so, for United States Government purposes. The Department of Energy will provide public access to these results of federally sponsored research in accordance with the DOE Public Access Plan (http:// energy.gov/downloads/doe-public-access-plan).
}

National Laboratory (ORNL) completed a resonance parameter evaluation of four tungsten isotopes in August 2014. The ${ }^{182,183,184,186} \mathrm{~W}$ resonance evaluations were submitted as an ENDF-compatible file to be part of the ENDF/B-VIII.0 nuclear data library [3].

Currently, the ENDF/B-VII.1 tungsten evaluations in the resolved resonance region (RRR) are up to $2.2 \mathrm{keV}$ $\left({ }^{183} \mathrm{~W}\right), 4.5 \mathrm{keV}\left({ }^{182} \mathrm{~W}\right), 4 \mathrm{keV}\left({ }^{184} \mathrm{~W}\right)$, and $8.5 \mathrm{keV}\left({ }^{186} \mathrm{~W}\right)$. These evaluations were performed by Leal [4] in the RRR and by Trkov et al. [4] in the unresolved resonance and fast region. The evaluations were tested on the basis of benchmark models taken from the SINBAD [5] and the ICSBEP [6] compilation. The number of benchmarks used in that analysis involved neutron flux spectra in the intermediate and fast energy range that are only slightly sensitive to the RRR. Therefore, the validation analysis of the Grenoble lead-slowing-down (LSD) benchmarks [7] whose experimental spectrum extends over the entire resolved resonance incident neutron energy range becomes an important test of the ORNL evaluations.

As shown in Table 1, in the analyzed energy range, the ORNL set of evaluations doubles the RRR energy range compared to the current library. The evaluation work follows the series of measurements on tungsten isotopes that was performed at the Geel Electron LINear Accelerator (GELINA) by Lampoudis in 2010 [8] and by Guber in 2012 [9]. The newly evaluated set of neutron resonance parameters, which is documented in three published works [10-12], considered the neutron and gamma exit channel $(c)$ for incident neutron partial $s$ - and $p$-wave to define the level energies $E_{\lambda}$, the probability amplitudes $\left(\gamma_{c}^{\lambda}\right)$ and the related partial widths $\left(\Gamma_{c}^{\lambda}\right)$. The analysis of the experimental data used the 
Table 1. Neutron energy range for the ORNL and ENDF/BVII.1 tungsten evaluations. The number of fitted levels of ORNL evaluations is also shown.

\begin{tabular}{lcc}
\hline Nucleus $\left(I^{\pi}\right)$ & $E_{\max }^{\text {ORNL }}\left(E_{\max }^{\mathrm{ENDF} / \mathrm{B}-\mathrm{VIII.1}}\right)$ & No. Levels \\
\hline${ }^{183} \mathrm{~W}\left(1 / 2^{-}\right)$ & $5(2.2) \mathrm{keV}$ & 387 \\
${ }^{182} \mathrm{~W}\left(0^{+}\right)$ & $10(5) \mathrm{keV}$ & 306 \\
${ }^{184} \mathrm{~W}\left(0^{+}\right)$ & $10(4) \mathrm{keV}$ & 178 \\
${ }^{186} \mathrm{~W}\left(0^{+}\right)$ & $10(8.3) \mathrm{keV}$ & 95 \\
\hline
\end{tabular}

SAMMY code [13], which performed a multi-level multichannel $R$-matrix fit to neutron data using the ReichMoore approximation. Experimental conditions such as resolution function, finite size of the sample, nonuniform thickness, and nuclide abundances of sample, multiple scattering, self-shielding, normalization, background, and Doppler broadening were also taken into account.

This paper presents a brief description of the Grenoble LSD benchmarks (Sect. 2) and discusses the results of the validation analysis (Sect. 3). Preliminary validation results were reported in 2013 [11] for the set of ${ }^{183} \mathrm{~W}$ preliminary resonance parameters included in the ENDF/B-VII.1 library. That analysis showed a good agreement with the experimental neutron spectrum but a relatively small impact of the new ${ }^{183} \mathrm{~W}$ resonance parameters that might be explained by the relatively homogeneous distribution of the isotopic tungsten, namely, ${ }^{182} \mathrm{~W}(26.5 \%)$, ${ }^{183} \mathrm{~W}(14.31 \%),{ }^{184} \mathrm{~W}(30.64 \%)$, and ${ }^{186} \mathrm{~W}(28.43 \%)$.

In the present validation work, the ORNL tungsten evaluations showed an improved agreement with the experimental response of the Grenoble LSD benchmark when they were included in the ENDF/B-VII.1 library. The improved agreement seems to be due not only to the interplay of different tungsten isotopes, but also to the extended resolved energy range as suggested by comparing the results from two sets of ORNL and JEFF3.2 evaluations. For the latter, the upper energy limits of tungsten RRR evaluations library $[14,15]$ are roughly the same as in the ENDF/B-VII.1 library.

\section{LSD benchmarks}

Perrot et al. [7] used an LSD spectrometer coupled to a specially designed pulsed neutron generator and new types of detectors with a modern data acquisition system to measure the $(\mathrm{n}, \gamma)$ and $(\mathrm{n}, \mathrm{f})$ cross sections of $\mathrm{Au}, \mathrm{Ag}, \mathrm{Ta}$, In, Th, U, Tc, Mo, Ni, Mn and W. The LSD spectrometer is a cubic assembly of 46.5 tons of pure lead $(99.99 \%)$ as shown in Fig. 1. The assembly consists of 8 cubic blocks with $80 \mathrm{~cm}$ sides.

A central channel allows the insertion of the accelerator glove finger. At the sides of the lead block, additional channels are used both for block handling and for detector insertion. Pure lead was chosen to ensure that impurities (mainly $\mathrm{Ag}, \mathrm{Bi}, \mathrm{Cd}, \mathrm{Cu}, \mathrm{Sb}$ and $\mathrm{Te}$ ) have a very small or negligible effect on the neutron flux and especially to reduce the photon production which strongly interferes with the $(\mathrm{n}, \gamma)$ reaction rate measurements in the irradiated samples. The lead block is shielded with a cadmium foil to prevent backscattering of slow neutrons. For that reason, measurements of rate of other reactions, e.g. fission rate, can be more accurate and reliable, however capture rates are also possible to deduce indirectly by subtracting the

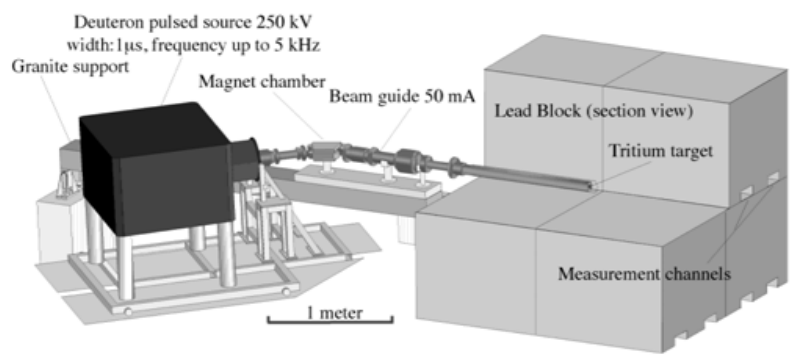

Figure 1. Diagram of GENEPI coupled to the LSD time spectrometer at ISN Grenoble (figure taken from Ref. [7]).

gamma background measured for a configuration without sample.

The neutron source, GEnerateur de Neutrons Pulsé Intense (GENEPI), consists of a duoplasmatron source producing fast $250 \mathrm{keV}$ deuteron pulses $<1 \mu$ s focused through a $5 \mathrm{~m}$ long tube (glove finger) onto a TiD or TiT target 1 . This pulse duration corresponds to the slowingdown time necessary for a $14.1 \mathrm{MeV}$ neutron to reach $100 \mathrm{keV}$. The nuclear reactions $\mathrm{D}(\mathrm{d}, \mathrm{n})^{3} \mathrm{He}$ or $\mathrm{T}(\mathrm{d}, \mathrm{n})^{4} \mathrm{He}$ produce neutrons with energies $2.67 \mathrm{MeV}$ and $14.1 \mathrm{MeV}$, respectively. Capture reaction rates were measured with a small Philips XP1911 $18.6 \mathrm{~mm}$ diameter photomultiplier coupled to a $\mathrm{CeF}_{3}$ scintillator. The time-dependent detector response after pulse was measured for empty irradiation channel (background) and samples of various sized and materials. For this paper, the $(\mathrm{n}, \gamma)$ measurement with the $1 \mathrm{~mm}$ thick disk made of natural tungsten is relevant.

In order to reproduce the measurements, a full-scale computational model was developed using MCNP [16]. The computational effort required to directly simulate the response of the detector was immense. This was due to the very large amount of the scattering events in the lead blocks relative to the number of neutrons reaching the sample, and also of the $\gamma$ photons produced in the sample and absorbed in the detector. Therefore, apart from implementing various variance reduction techniques, it was assumed that the capture rate in the sample is proportional to the response of the detector with the sample corrected by the background. Thus, the main assumption of the MCNP model is that the neutron sensitivity of the detector and $\gamma$ absorption in the sample are negligible. After validation of the computational model against the experimental values using various samples $(\mathrm{Mn}, \mathrm{Ni}, \mathrm{Au}$, $\mathrm{W}$, etc.), the model can be used for comparison and validation of cross sections from different nuclear data libraries.

Still, $10^{10}$ neutrons and more than 18000 minutes using 128 processors (2 Xeon E5450 quadcore, $3.00 \mathrm{GHz}$ ) were needed to complete each individual calculation and to achieve reasonable statistical uncertainty. The results from the calculations were first shifted in time to take into account the time at which the emission of neutrons occurs relative to the trigger signal of the pulse at the source. Then the results were converted to equivalent energy. It is important to note that the energy, equivalent to a given time after pulse, does not exactly correspond to the incident neutron energy. It corresponds to the effective energy at the given time, but the neutron energy distribution is broadened around that value. Although the energy scale is not exactly right, the comparison 


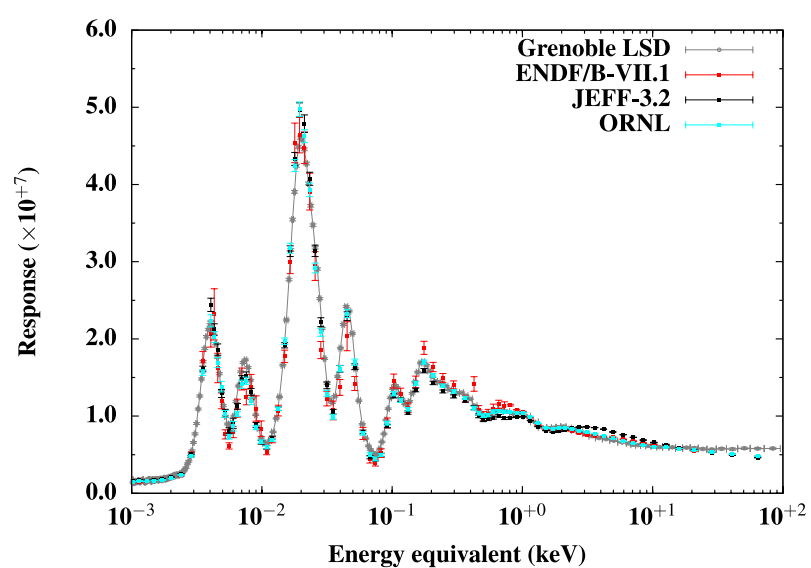

Figure 2. Experimental spectrum of the Grenoble LSD benchmark (gray dots) in the energy range of $1 \mathrm{eV}$ up to $100 \mathrm{keV}$ compared with the spectra obtained with the ENDF/B-VII.1 (red dots), JEFF-3.2 (black dots) and the set of tungsten ORNL evaluations (cyan dots).

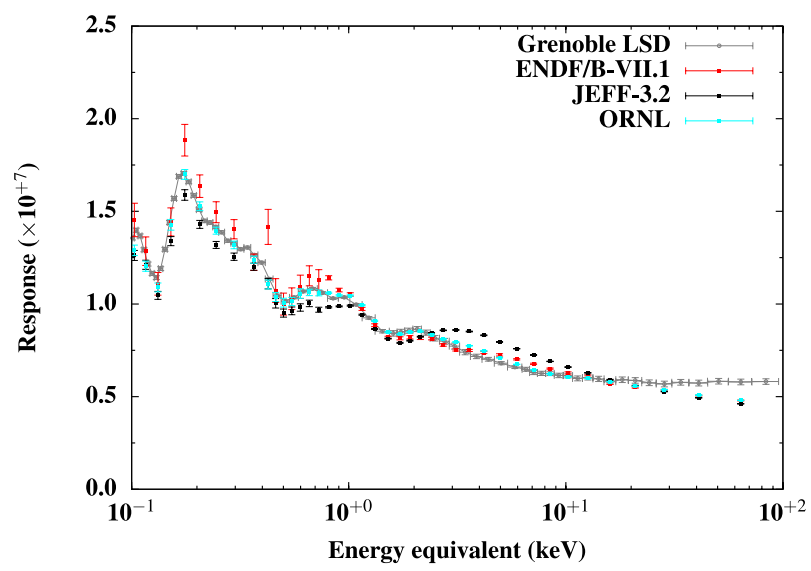

Figure 3. Magnification of Fig. 2 in the energy range of $100 \mathrm{eV}$ and $100 \mathrm{keV}$.

between measurements and calculations is meaningful because the same transformation is applied exactly to the measured data and to the calculated values. The main results observed for the tungsten sample, are presented in the following section.

\section{Results}

Together with the ENDF/B-VII.1 nuclear data library, the recently released ORNL tungsten evaluations in the RRR were used in MCNP [16] to calculate the reaction rate spectra of the Grenoble LSD benchmark model. Figure 2 and the corresponding magnification, Fig. 3, show the obtained spectrum (cyan dots) in comparison to the experimental spectrum of the Grenoble LSD benchmark (gray dots) in the energy range from $1 \mathrm{eV}$ to $100 \mathrm{keV}$. The overall behavior of the evaluated spectrum up to $10 \mathrm{keV}$ is in good agreement with the experimental data, and most importantly, an improved agreement can be seen with respect to the current tungsten evaluations in the ENDF/BVII.1 library. The most remarkable improvement resides in the $100 \mathrm{eV}$ up to $10 \mathrm{keV}$ energy range, which is clearly visible in Fig. 3. For energies above $20 \mathrm{keV}$ the reaction rate seems to under predict the experimental data which are

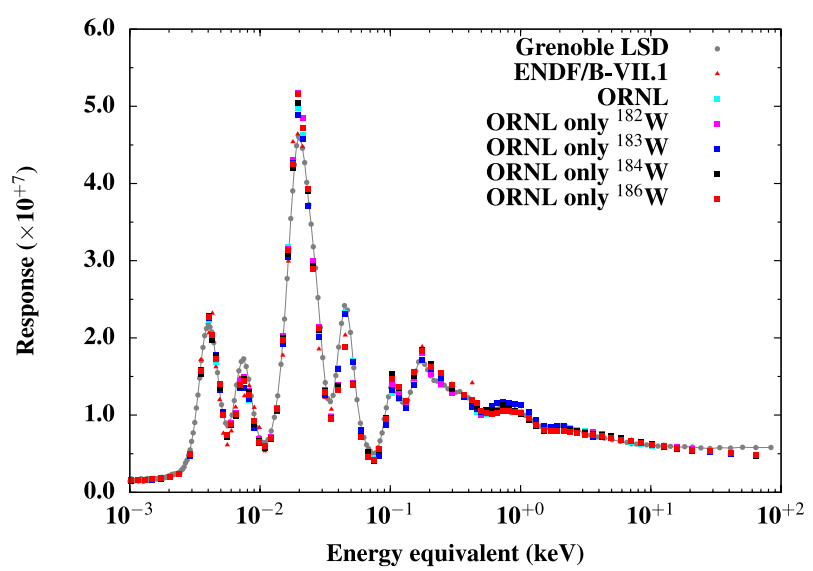

Figure 4. Experimental spectrum of the Grenoble LSD benchmark (gray dots) in the energy range of $1 \mathrm{eV}$ and $100 \mathrm{keV}$ compared with the spectra obtained with the ENDF/B-VII.1 (red dots), and the set of tungsten ORNL evaluations (cyan dots). The interplay of different tungsten isotopes is also shown.

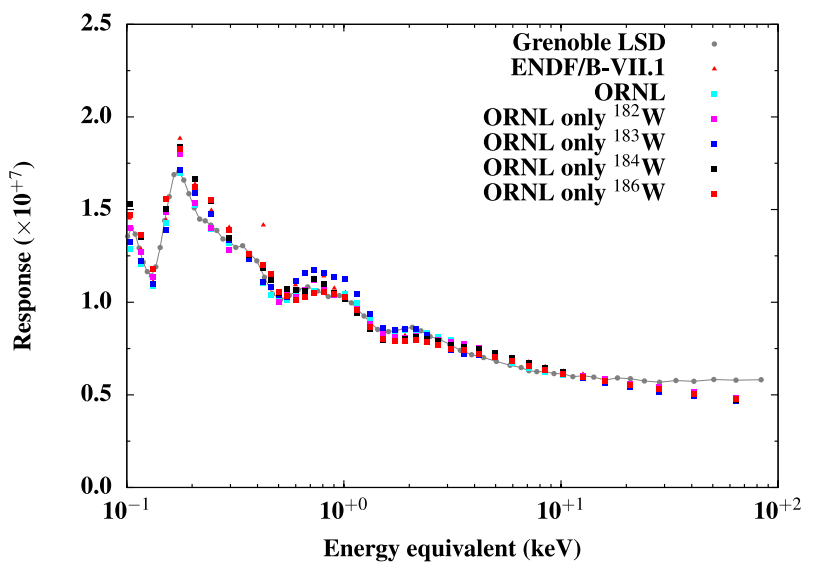

Figure 5. Magnification of Fig. 4 in the energy range of $100 \mathrm{eV}$ and $100 \mathrm{keV}$.

possibly affected, in this energy range, by a systematic over prediction of the reaction rate, as the same trend was found for other isotopes such as manganese. The overprediction at energies close to $100 \mathrm{keV}$ might be due to a lack of information on neutron pulse shape and duration.

Figures 2 and 3 also display the results from JEFF3.2 library (black dots). Although in good agreement with the experimental spectrum and comparable to the ORNL results (cyan dots) up to $100 \mathrm{eV}$, the results from JEFF3.2 evaluated data are clearly in poor agreement in the energy range between $100 \mathrm{eV}$ up to $10 \mathrm{keV}$. One possible explanation for the JEFF performance could be the limited energy upper range of the RRR evaluations in the JEFF-3.2 nuclear data library (similar to ENDF/B-VII.1 library).

Due to the relatively homogeneous distribution of the isotopic tungsten, it was also important to quantify the impact of each tungsten evaluation on the calculated reaction rate spectrum. In Fig. 4 and in the corresponding magnification in Fig. 5, the interplay of the four tungsten isotopes obtained by including each evaluation individually in the ENDF/B-VII.1 library is shown. Although each component contributes to some variation in the calculated spectrum, the ${ }^{183} \mathrm{~W}$ isotope (square blue 
dots) plays a large role in predicting the reaction rate in the $100 \mathrm{eV}$ to $2 \mathrm{keV}$ energy range, at least in relation to the ENDF/B-VII.1 evaluations. As in the preliminary validation analysis [11], the impact of the ${ }^{183} \mathrm{~W}$ isotope is also confirmed in the present work.

The results from the selected nuclear data libraries are displayed in Figs. 2 and 3 with the uncertainties corresponding to the number of neutron histories used in the Monte Carlo simulations. In this regard, we believe the large statistical uncertainties for the ENDF/B-VII.1 library do not affect the final conclusions and, for the future, we plan to perform similar analyses within the next release of the US ENDF nuclear data library, i.e., ENDF/B-VIII.0.

\section{Conclusion}

Following the ORNL evaluation work on tungsten isotopes recently included in the $\beta$ releases of the ENDF/B-VIII.0 library, a validation analysis on the basis of the Grenoble LSD benchmark model was performed in the energy range between $1 \mathrm{eV}$ and $100 \mathrm{keV}$.

The calculated reaction rate spectrum showed overall good agreement with the experimental spectrum, and remarkable improvements with respect to the evaluated tungsten data from ENDF/B-VII.1 and JEFF-3.2 libraries were seen mainly in the energy range between $100 \mathrm{eV}$ up to $2 \mathrm{keV}$. The improved agreement seems to be due to the interplay of different tungsten isotopes and also to the extended resolved energy range as suggested by comparing the results from two sets of ORNL and JEFF3.2 evaluations.

Future work will include a complete and energetically comprehensive suite of validation tests within the ENDF/B-VIII.0 nuclear data library. Also, this would be extremely important in validating the considerable change in the evaluated scattering thermal cross section for ${ }^{183} \mathrm{~W}$ isotope on the basis of benchmarks sensitive to the scattering cross sections at thermal energies.

This work was supported by the US Department of Energy (DOE), Nuclear Criticality Safety Program (NCSP) funded and managed by the National Nuclear Security Administration for DOE.

\section{References}

[1] M.B. Chadwick et al., Nuclear Data Sheets 107, 2931 (2006)

[2] M.B. Chadwick et al., Nuclear Data Sheets 112, 2887 (2011)

[3] D.A. Brown, ND2016 Conference Proceedings (to be published)

[4] A. Trkov et al., Nuclear Data Sheets 112, 3098 (2011)

[5] P. Batistoni et al., J. Nucl. Materials 329-333, 683 (2004)

[6] ICSBEP 2006, NEA/NSC/DOC(95)03 (2006)

[7] L. Perrot, Nucl. Sci. Eng. 144, 142 (2003)

[8] C. Lampoudis et al., J. Korean Phys. Soc 59, 1860 (2011)

[9] K.H. Guber, Private Communication (2012)

[10] M.T. Pigni et al., PHYSOR 2012 Conference Proceedings (2012)

[11] M.T. Pigni et al., Nuclear Data Sheets 118, 147 (2014)

[12] M.T. Pigni et al., ICNC 2015 Conference Proceedings (2015)

[13] N.M. Larson, Report ORNL/TM-9179/R8 (2008)

[14] I. Sirakov et al., JRC 78706 (2013)

[15] F. Emiliani et al., JRC 88008 (2013)

[16] J.T. Goorley et al., Nuclear Technology 180(3), 298 (2012) 